\title{
Laser beam scattering for the detection of flat, curved, smooth, and rough microplastics in water
}

\author{
Benjamin O. Asamoah ${ }^{1} \cdot$ James Amoani $^{1} \cdot$ Matthieu Roussey $^{1} \cdot$ Kai-Erik Peiponen ${ }^{1}$
}

Received: 30 September 2019 / Accepted: 13 January 2020 / Published online: 3 February 2020

(c) The Author(s) 2020

\begin{abstract}
Microplastic (MP) pollution is alarming and poses an imminent threat to the environment with a direct impact on our health and that of fauna in natural water bodies. The understanding of light-MP interactions in water as well as the need for low-cost and robust optical sensors for the detection of MPs that appear everywhere is, therefore, necessary. We have demonstrated the use of a prototype optical sensor in the detection of flat and curved [from polyethylene terephthalate (PET) water bottle] pristine and rough MPs from commercial PET and low-density polyethylene plastics in water. The optical sensor utilizes a photodiode and charge-coupled device (CCD) camera to record simultaneously the specular reflection and the speckle pattern modified by the MPs. In this study, we have exploited the specular reflection in the detection of the pristine samples, whereas the speckle contrast, the normalized standard deviation of the speckle pattern intensity, is utilized in the qualitative estimation of the "effective" surface roughness of the MPs. With the sensor, one can, therefore, detect PET MPs with varying average surface roughness, $\mathrm{Ra}$ an indication of MP aging, embedded in water. The prototype can detect the effect of size, type, curvature, transparency, and the translucency of sunken and/or floating MPs in water based on the reflection, scattering, and the (edge) diffraction of light. However, the optical sensor is limited in the discrimination of MP concentration in water. Further modifications to the sensor are needed for its practical implementation in complex natural water bodies and wastewaters.
\end{abstract}

Keywords Microplastics $\cdot$ Surface roughness $\cdot$ Specular reflection $\cdot$ Laser speckle pattern $\cdot$ Speckle contrast

\section{Introduction}

There is a growing interest in the investigation of microplastic and its pollution in recent times. In the past 3 years, over 1000 publications [1] have been dedicated to the subject demonstrating the unique interest and perhaps the evasiveness of the problem. Part of the reasons that have piqued

Benjamin O. Asamoah

Benjamin.asamoah@uef.fi

Kai-Erik Peiponen

kai.peiponen@uef.fi

James Amoani

amoani.james@yahoo.com

Matthieu Roussey

matthieu.roussey@uef.fi

1 Department of Physics and Mathematics, University of Eastern Finland, P.O. Box 111, 80101 Joensuu, Finland the interest of researchers and policymakers are the demonstrated direct impact of microplastic pollution on the environment [2] such as in aquatic life and on human health [1]. For example, MPs have been identified in agricultural soil [3] and in fish [4], which are for direct human consumption.

Plastics that pollute both natural water bodies and municipal wastewater systems are a subject of mechanical erosion [5]. Such an erosion causes cracks and surface (micro)roughness of the microplastics, and this surface roughness is a direct measure of the aging of the microplastic [6]. Additionally, surface properties of plastics and MPs are indicative of the degradation process [7]. Surface roughness in addition to air pockets offers a platform for the adsorption of organic and inorganic contamination particles [8-10] that may have lower or higher gravity than that of the MP. Additionally, surface roughness has a consequence on the hydrodynamic properties of MPs, namely on the drifting of MPs over long distances [11]. Although the surface roughness is insignificant for nanoplastics, it becomes a relevant quantity for MP 
with a size of ca $5 \times 5 \mathrm{~mm}^{2}$ that is abundant in marine surface waters [12]. Unfortunately, FTIR and Raman spectroscopies that have been used to identify MPs do not provide in situ information on the surface roughness of the MP. This has motivated us to investigate the possibility to detect rough MPs with a different surface roughness on both sides under laboratory conditions. Note that in reality, rough MPs may be smooth and transparent at the initial stages of pollution. For example, a partially filled or empty PET bottle that is floating over water first experiences roughening of the outer envelope surface. Later on, smaller particles arising from long erosion processes will result in the two surfaces having a different magnitude of roughness due to the aging and degradation process. In this paper, we have simulated the erosion process of surface roughening by sand and investigated MPs with rough surfaces on both sides. We applied laser speckle pattern contrast analysis (LASCA) as a qualitative estimation of the effective surface roughness of the MPs. Moreover, we also employed the specular reflection of a laser beam of a robust and portable prototype [13] to detect two different plastic types as well as flat and curved pristine samples. We propose that such a simple method is promising regarding monitoring of aging and hence contamination issues of MPs by detecting the laser light scattering from rough and smooth MPs.

\section{Materials and methods}

We have studied flat MPs from commercial polyethylene terephthalate (PET) and slightly translucent low-density polyethylene (LDPE) plastic types (Goodfellow, UK), and curved PET from a water bottle. An approximate size of $5 \times 5 \mathrm{~mm}^{2}$, representing the upper limit of the microplastic size definition [14], was considered in the present study as similar MP sizes are readily created by weathering in water [15]. Both pristine $(\mathrm{Ra}<0.06 \mu \mathrm{m})$ and roughened PET MPs as well as pristine LDPE $(\mathrm{Ra}<0.10 \mu \mathrm{m})$ were considered. The latter is interesting, because it shows some volume inhomogeneity. To prepare the rough MPs, larger samples of the pristine PET were first roughened on both sides with different sandpaper grits and cut into the required size. The cutting of the MPs from the larger plastic sheets may result in flat samples with slightly curved edges. The samples were then grouped, such that each group has the same average roughness one surface of the $\mathrm{MP}$, e.g., $\mathrm{Ra}=0.33 \mu \mathrm{m}$, but different surface roughness on the other surfaces. The groups are accordingly referred to as, using the grit size, G80D, G320D, and G600D, as an example, indicating that the constant average surface roughness (in each group) was achieved with grits values of 80,320 , and 600, respectively. Moreover, the naming convention also means that the light was incident on the surface with constant roughness.
The refractive index of the commercial samples obtained from ellipsometric measurements [16], the sandpaper grit values, and the corresponding average surface roughness measured from five scanned lines with Mitutoyo stylus profilometer (SJ-210, Japan) are shown in Table 1. Figure 1a also shows schematics of the roughened flat PET MPs, with different average (effective) surface roughness on side 1 and 2, and curved MPs from the PET bottle (B). The curved (semi-cylindrical) PET bottle has a diameter and thickness of $6.3 \mathrm{~cm}$ and $0.4 \mathrm{~mm}$, respectively.

As examples, the roughness profiles for G1200, G600, and G320 samples, and the roughness distribution from the profiles, obtained from a more sensitive profilometer, Dektak150 (Veeco Instruments, B.V), than the Mitutoyo are, respectively, shown in Fig. 1b, c. The corresponding fitted Gaussian distributions of the roughness are also shown in the same figure. The Mitutoyo profilometer exploits a Gaussian height distribution in the estimation of the average roughness, and hence, it was not possible to directly obtain the surface roughness profiles from such an inexpensive device. However, it is a nice alternative to other high-end devices for roughness determination. From Table 1 and Fig. 1b, c, the obtained roughness values from both profilometers are in agreement for the two samples and the Gaussian assumption by the Mitutoyo profilometer is also validated.

The optical sensor is a combination of a portable commercial handheld gloss meter (MGM device, Finland), for measuring the specular reflection, and an attached chargecoupled device (CCD) camera (DCC1465C-HQ, Thorlabs), for capturing the speckle pattern [17]. Both components are connected to a laptop computer to form a single optical device for reflection and transmission measurements as presented in this study. The recorded specular reflection signal is within the accuracy of $c a .0 .10 \%$. A detailed description of the portable optical sensor is described in [13]. However, we briefly state some specific features. Its superior advantage is in the use of a diffractive optical element (DOE) [18], with

Table 1 The refractive index $(n)$, the sandpaper grit, and the respective average surface roughness ( $\mathrm{Ra}$ ) of the samples

\begin{tabular}{llll}
\hline Sample & $\begin{array}{l}\text { Refractive index }(n) \\
\text { at } 635 \mathrm{~nm}\end{array}$ & Grit & $\begin{array}{l}\text { Avg. surface } \\
\text { roughness, Ra } \\
(\mu \mathrm{m})\end{array}$ \\
\hline $\begin{array}{l}\text { Polyethylene } \\
\text { terephthalate } \\
\text { (PET) }\end{array}$ & 1.5570 & 1200 & $0.33 \pm 0.04$ \\
& & 600 & $0.63 \pm 0.08$ \\
& & 320 & $1.15 \pm 0.17$ \\
& 180 & $2.52 \pm 0.20$ \\
& & 80 & $4.12 \pm 0.25$ \\
$\begin{array}{c}\text { Low-density PE } \\
\text { (LDPE) }\end{array}$ & 1.4920 & - & $<0.10$ \\
\hline
\end{tabular}



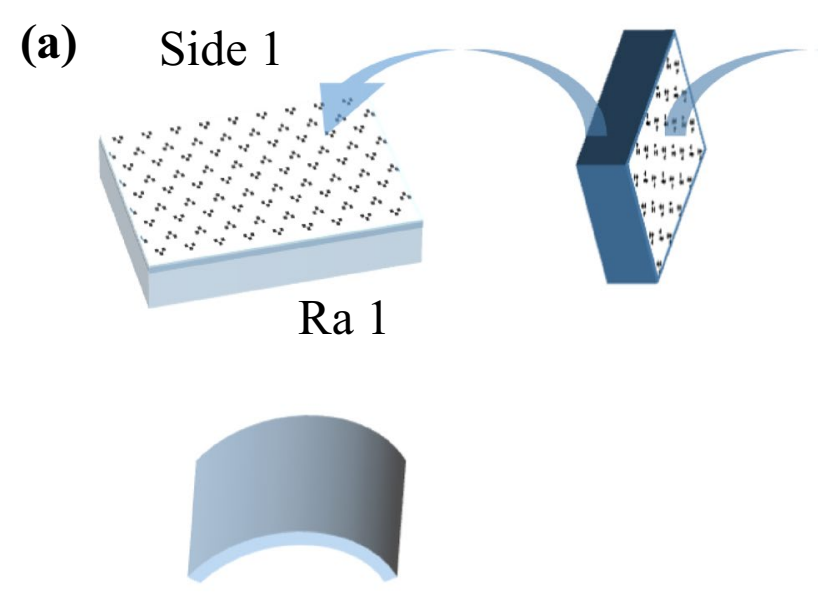

\section{B MP Concave}
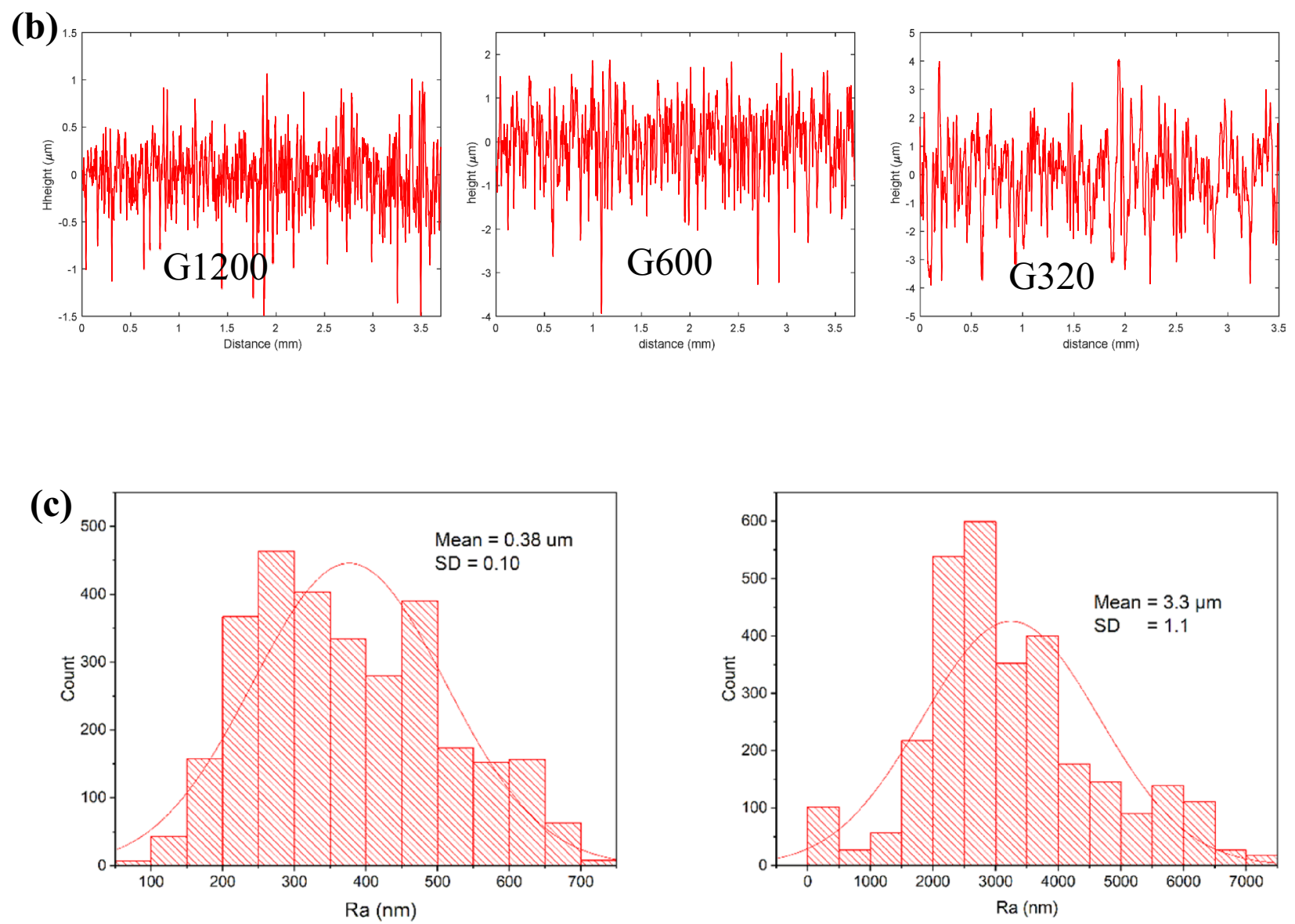

Fig. 1 a Schematics of the roughened PET MPs and pristine MPs from a bottle (B), sides 1 and 2 have different average surface roughness, Ra 1 and $\mathrm{Ra} 2$, respectively. b Roughness profiles for G1200, G600, and G320, and c the roughness distribution for G1200 and G80 samples

focal length and aperture size of $20 \mathrm{~mm}$ and $2 \mathrm{~mm} \times 2 \mathrm{~mm}$, respectively, to uniquely filter the diffusely reflected light to obtain only the specular component. The light source is a $0.8 \mathrm{~mW}$ stable semiconductor laser $(635 \mathrm{~nm})$, which gives regular interference fringes for smooth MPs on the chip of a
Side 2

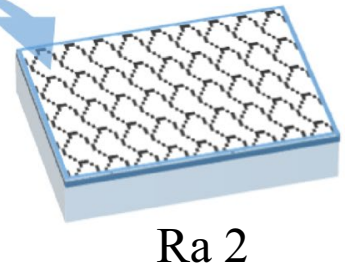

B MP Convex 
propagating light beam interacting with the MPs is diverging. In the present configuration, the detection plane of the CCD camera was positioned $11 \mathrm{~cm}$ from the glass disk. The operating principles of the optical sensor are based on reflection and forward scattering is illustrated in Fig. 2. The angles of incidence and detection are $6^{\circ}$ from the normal.

The volume compartment was filled with $700 \mu$ of freshwater (FW), from the Lake of Pyhäselkä (Joensuu, Finland), which typically contains algae and other micro-organisms, to a height of $2.8 \mathrm{~mm}$, and the samples were added. The MPs were studied with a glass disk having one of its surfaces roughened. In this case, with the coherent light source, a speckle pattern, which is a grainy structure of bright and dark spots, is formed at the detection plane of the CCD camera. By placing the samples in the water, the light-MP interaction further modifies the initially generated speckle pattern. Considering the originally generated speckle pattern from the rough glass disk, we can consider the speckle pattern recorded in the detection plane of the CCD camera as a form of speckled speckle [19]. Although the pristine, flat, curved, or rough MPs differently modifies the initially generated speckle pattern by the rough glass disk, we simply refer to the obtained images on CCD as a speckle pattern. The use of speckle pattern has found many practical applications [20-23]. The method of speckle pattern projection [20] has been suggested for the detection of the thickness of transparent objects. Similarly, we exploit the speckle pattern projection technique in this paper but for the novel application of sensing the surface roughness of MPs.

The speckle pattern formed by the rough glass (surface) is due to the random interference of the coherent light field as a result of the random phase. From the prototype, we simultaneously obtain the specular reflection in the reflection mode and the speckle pattern in the transmission mode. In the

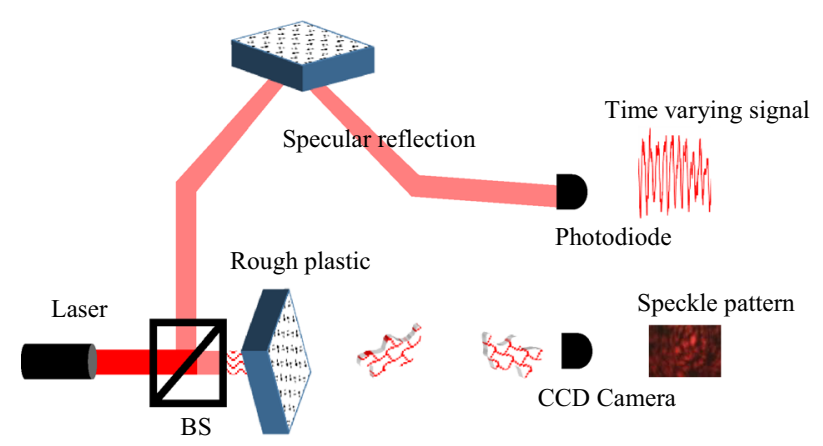

Fig. 2 Operating principle of the optical sensor. The handheld sensor detects the time-varying specular reflection, whereas the charge-coupled device (CCD) detects the transmitted light signal from smooth MPs or the speckle pattern from the rough MPs. The beam splitter (BS) in the schematics is for illustrative purposes only and for visual clarity on the operating principles. For further details on the schematics, we refer the reader to Fig. 1 of [13] detection plane of the CCD camera, the speckle pattern can be seen to be non-stationary. It is, therefore, generally treated as a statistical phenomenon. Hence, it is usually quantified using the speckle contrast $(C)$ which is the normalized standard deviation expressed in Eq. (1) [17]:

$C=\frac{\left[\left\langle I^{2}\right\rangle-\langle I\rangle^{2}\right]^{1 / 2}}{\langle I\rangle}$,

where $I$ is the light intensity and $\langle\cdots\rangle$ is the ensemble average. $C$ has a minimum and maximum value of 0 and 1 for undeveloped and fully developed speckles, respectively. $C$ also depends on the measurement configuration or conditions such as the detection method, the wavelength of the light source, the angle of incidence, as well as the properties of the incident light [5]. The recorded speckle pattern was corrected by subtracting the measured stray light from the background and the speckle contrast estimated from the red channel of the $1280 \times 1024$-pixel intensity distribution. The results averaged from three (3) sets of measurements are shown in the next section for both the specular and the estimated speckle contrast values, with the error bars, for the different samples.

\section{Results and discussion}

Larger plastics can be transformed into MPs upon interacting with the harsh aquatic environment. These interactions may affect the MP surface quality by turning initially smooth ones into rough MPs, as well as the curvature of the MPs. Under controlled laboratory conditions, we have examined the role of surface roughness, which correlates with the aging and degradation, on both surfaces of MPs, and the curvature on the optical detection of such MPs in water with the portable optical sensor. The sensor records simultaneously the specular reflection (from pristine samples) and speckle pattern from which the quantitative speckle contrast $(C)$ is determined.

Figure $3 \mathrm{a}$ shows the mean of the time-dependent specular reflection signals of the pristine samples. As an example, the actual time dependence of the signal is shown for only one of the PET samples. The specular reflection depends on both the refractive index (RI) mismatch between the MPs and water (at the probe wavelength), and the intensity of the incident light. From Fig. 3a, we observe that the PET gives a higher average reflection signal than the freshwater (FW). On the contrary, for LDPE, with slight volume inhomogeneity, we obtain a lower average reflection signal as it is likely to scatter more of the light than the transparent PET MP. These two observations show that a foreign material with a different refractive index than that of water is 
Fig. 3 a Specular reflection from the pristine samples and water (FW). Polyethylene terephthalate (PET), lowdensity polyethylene (LDPE), and curved samples from PET bottles (B): concave and convex shapes. The signal from PET, as an example, shows the time dependence of the specular reflection. b Speckle patterns of water (FW) and pristine MPs of PET, LDPE, and PET bottle (B MPs) with that of water subtracted. Convex and Concave. The last four figures are slightly enhanced for easy visualization $\mathbf{a}$

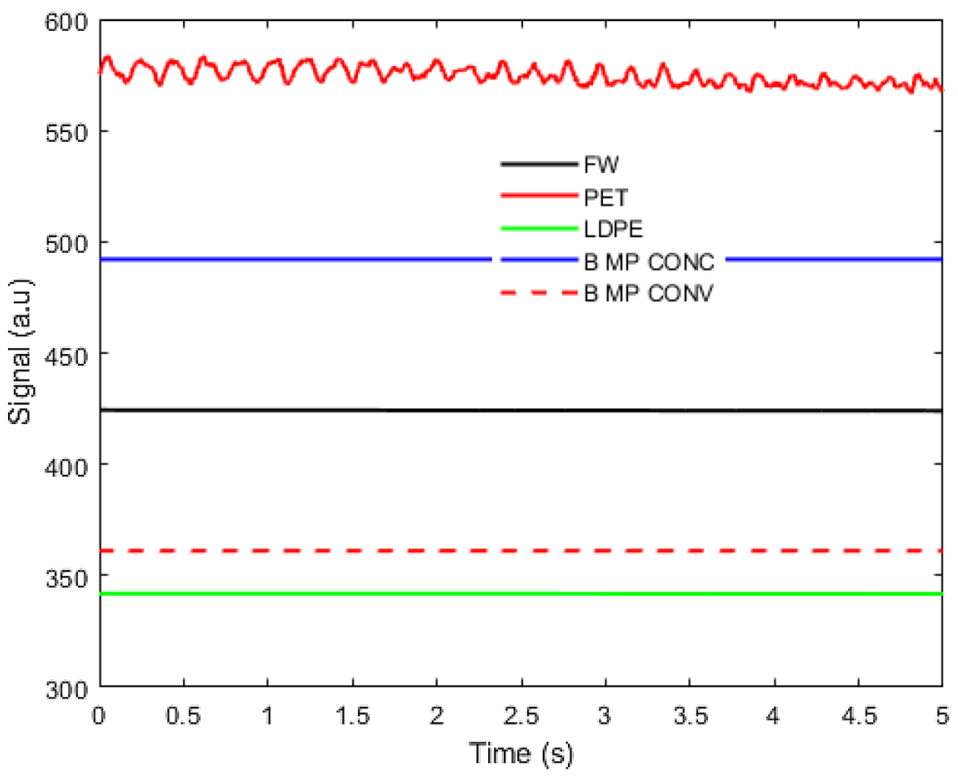

b

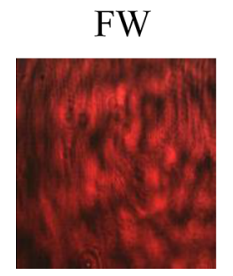

PET

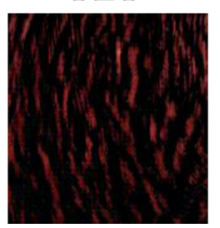

LDPE

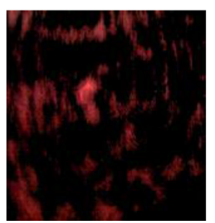

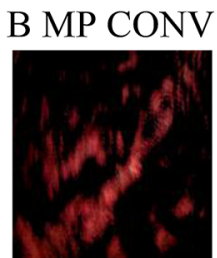

B MP CONC

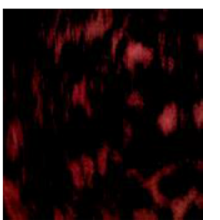

present. For the PET bottle MPs, the B MP CONC, with the inner curvature towards the incident light, shows a higher signal than the B MP CONV that has the outer curvature towards the incident light. It is worth to note that the B MP CONC is the same sample as B MP CONV that was placed differently with respect to the incident light source. Thus, the B MP CONC tends to act as a converging mirror focusing the reflected light, whilst the B MP CONV, on the other hand, diverges the incident light beam. Therefore, one can conclude that by measuring the specular component of the reflected light, the optical sensor can distinguish the different types of MPs as well as their planarity. This is rather the striking feature of the sensitivity of the DOE to detect such variations in the specular reflections.

We note that natural water bodies, where the fully developed sensor is intended for use, are complex media containing micro-organisms such as algae and other micro-particles. The concentration of MPs in these media can also be very low and the other particles can contribute to the scattering of the light. However, the prototype sensor can, nonetheless, be a viable option for the discrimination of the MPs from the other particles. The refractive index contrast between water and algae is lower (ca. 1.06 \pm 0.04 ) [24] than that of water and plastics (ca. $1.17 \pm 0.01$ ) at the probe wavelength of the laser light source used. The difference in the refractive index contrast will result in the different reflection coefficient of the specular reflection allowing the identification of the different particles. Moreover, the presence and movement of different minerals can increase the complexity of natural water bodies. In such a noisy background, one can perform signal processing based on a triple correlation [25] of the specular reflection signal and the speckle pattern (in Figs. 3b and 4). This process requires the correlation of three different signals sampled at three different time instances to improve the signal from the MPs. However, such signal processing was not necessary in the present study as the freshwater showed no background noise.

Figure $3 b$ shows the speckle patterns for the pristine MPs with that of water subtracted. The subtraction is done to qualitatively examine the extent to which the samples modify the originally generated speckle pattern from the rough glass disk. However, this subtraction is not necessary when the sensor is implemented in real water bodies. From the figure, we observe the different modifications of the speckle patterns by the pristine MPs. The PET, in comparison to the FW, shows an almost dark pattern as expected from a transparent material, whereas the LDPE shows a much brighter pattern. Interestingly, for the B MP (CONC and CONV), which is the same MP, we observe clearly how the curvature contributes to the significant 


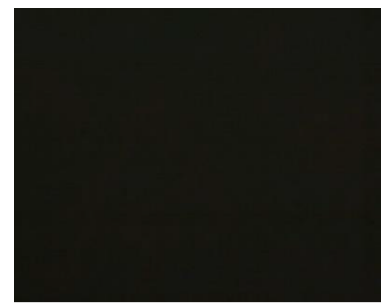

G80D

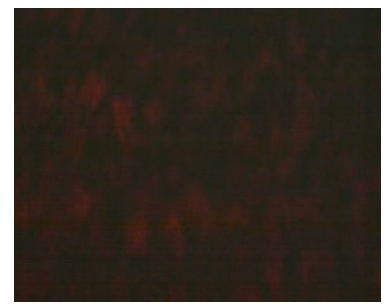

G600D

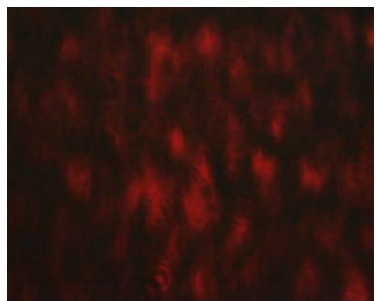

G180D

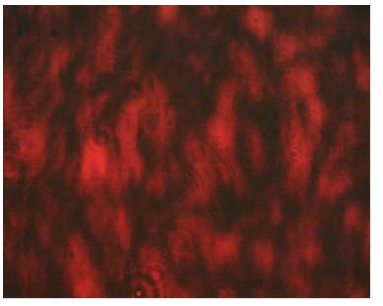

G1200D
Fig. 4 Recorded speckle pattern for PET MPs with varying average surface roughness (G80D-G1200D) on the lower surface (towards the incident light) and constant average roughness (G320) on the upper surface

modification of the speckle pattern. Since the pristine MPs, with little or no roughness, practically transmit the incident speckle pattern, we do not use the speckle contrast to estimate the modification to the initially generated speckle pattern.

In Fig. 4, we further show the speckle patterns for the case of flat PET MPs, with roughness on both surfaces, recorded at the detection plane of the CCD camera. Here, we consider the case where the top surface has an average roughness of $1.15 \mu \mathrm{m}(\mathrm{G} 320)$ and that of the bottom surface, to which the probe light is incident, varies from 0.33 to $4.12 \mu \mathrm{m}$ (G1200D-G80D). The surface roughness, as well as the properties of the probing light such as the coherence and beam waist, are known to influence the size and density of the speckle pattern [26]. The G80D, with the highest average surface roughness, shows the darkest pattern whereas the G1200D shows the brightest speckle pattern. Following this observation and keeping the average roughness constant on the top surface, the brightness of the speckle pattern was expected to increase with decreasing average roughness on the bottom. However, G180D has brightness higher than that of G600D. Such an irregularity may be apparent in the speckle contrast as it is directly obtained from the patterns. We note that due to the complex nature of the samples, namely having different roughness on the surface, the speckle pattern modification by the different rough surfaces can also be convoluted. For such a relatively small thickness of the PET MPs ( $0.25 \mathrm{~mm}$ of originally smooth MPs), the incident light may encounter a surface with an effective roughness, $\Delta n \mathrm{Ra}$, which is the combination of the roughness
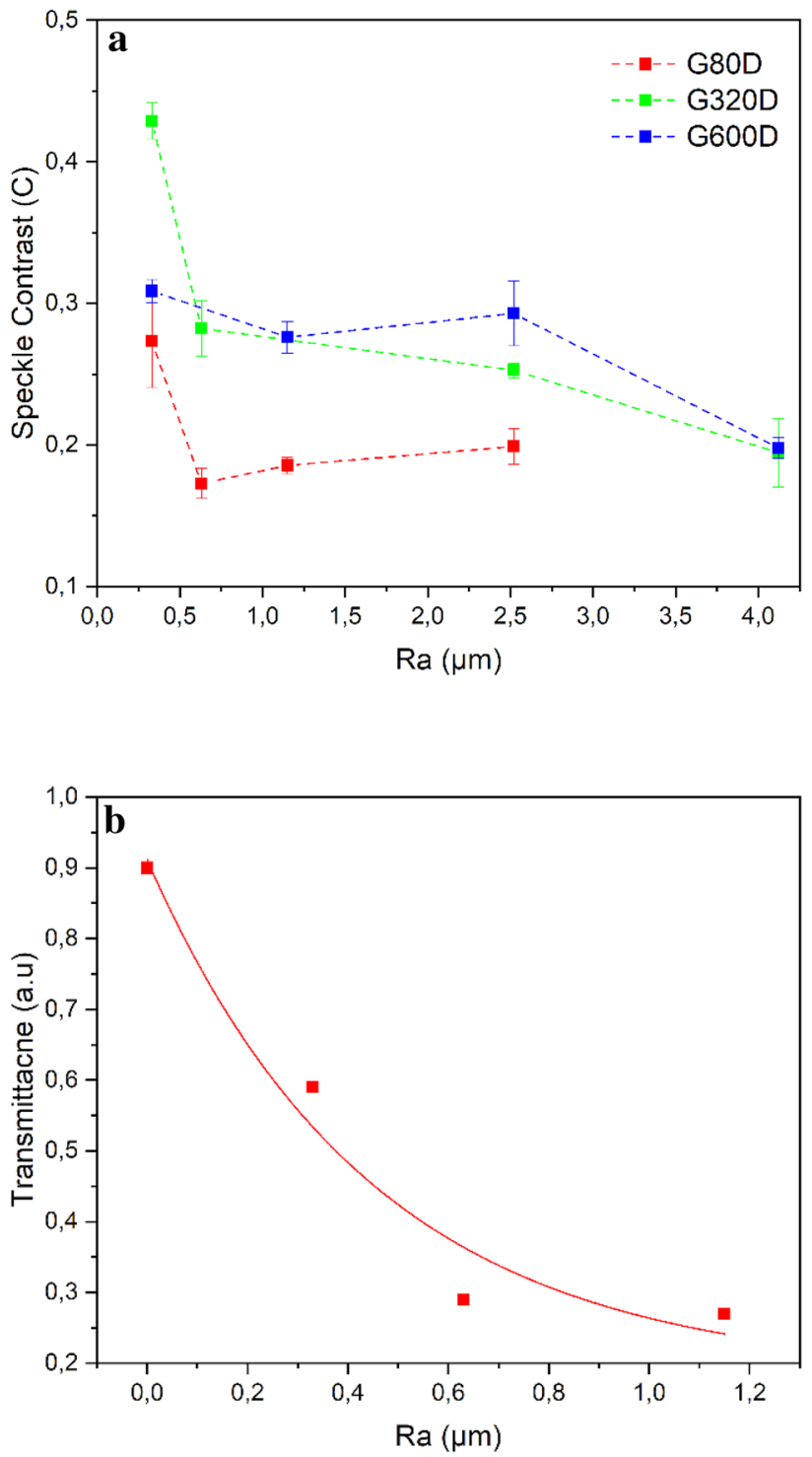

Fig. 5 a Speckle contrast of both sided roughened PET MPs. The speckle contrast decreases with increasing surface roughness on both the top and bottom surfaces of the MPs. The legend (G80D) shows the surface on which the laser light is incident. b Transmittance of thin sheets of roughened samples at $635 \mathrm{~nm}$. The transmittance decreases exponentially with the average surface roughness

on both surfaces where $\Delta n$ is the RI difference of the MP and the ambient medium. This effective roughness can vary regarding the manual method of sandpapering.

The speckle contrast, $C$, is calculated from the recorded speckle patterns using Eq. (1). We note that the sample with the largest effective roughness is within the limit of the validity of $C$ for the conventional case of flat samples. However, for the flat MPs with possible slightly curved edges, such as in this case, their behavior, in general, tends to be rather complex. As an example, we show, in Fig. 5a, how the average roughness on the top surface, with reference to 
the incident light source, influences light and MP interaction for each sample group with the same average surface roughness on the bottom surface. The speckle contrast, with the error bars, presented in Fig. 5a is a promising tool in the detection of MPs in water based on the average surface roughness of the MPs. We observe that the speckle contrast decreases with increasing top surface roughness for each specific bottom surface roughness. Furthermore, decreasing the average surface roughness of the bottom surface correspondingly increases the speckle contrast especially for roughness equal to or larger than the wavelength of the incident light source. The abnormality namely G600D having lower speckle contrast than G320D, with effectively the same top surface roughness, is due to the variation in the speckle pattern as explained above. The decreasing of the contrast values with increasing surface (top or bottom) roughness is counterintuitive. Conventionally, speckle contrast is known to positively correlate with effective surface roughness [26, 27] which is the product of the $R a$ and the difference in the refractive index of the sample and that of the environment. However, we wish to note that, in such studies, the samples have been illuminated with either collimated [27, 28] or focused [26] beams, whereas we have used a diverging speckle pattern illumination [21]. To further verify the counterintuitive trend and the influence of the surface roughness on the speckle intensity, the transmittance of thin sheets of smooth and roughened PET samples in water was measured at $635 \mathrm{~nm}$ with a spectrophotometer. The result is shown in Fig. 5b. We observe an exponential decrease in the transmittance with an increase in the "effective" surface roughness on both surfaces of the MPs. In such a situation, the transmittance can be related to the roughness, $\mathrm{Ra}$, as $T=\mathrm{To}+A \exp (-\alpha \mathrm{Ra})$. To represent the transmittance of the surrounding medium (in the absence of the plastic) which is equivalent to 1 for water in this case; $A$ and $\alpha$ are, respectively, the fitting parameter and the effective absorption coefficient of the plastic in water, since water does not absorb the specified wavelength. For smooth MPs, $\mathrm{Ra}=0$, the transmittance is, therefore, dominated by the intrinsic absorption of the PET sheets in water.

The transmittance curve dictates that by simply increasing the average surface roughness, we decrease the amount of light reaching the detector of the spectrophotometer which can be due to the increased scattering as well as reabsorption of the scattered photons by the plastic sample. Analogously, we can also conclude that the interaction of the diverging incident beam with the rough MPs reduces the light intensity radiated in the detection space of the CCD camera. Thus, the use of the diverging beam, and the observed increase in scattering and (re)absorption due to increased roughness, as explained above, affects the speckle pattern and its corresponding speckle contrast. In future studies, we also wish to explore the speckle correlation in the investigation of MPs in water.

\section{Conclusion}

In the natural environment, MPs resulting from primary and secondary sources interact with the harsh conditions leading to the degrading of the surface quality. Such degradation can result in the time-dependent roughening of the MPs forming a suitable habitat for micro-organism and contaminants, and also affecting the hydrodynamic properties as well as the distribution of the MPs. In this work, we have examined the role of different average surface roughness, which has a direct correlation on the aging of MP, on the optical detection in freshwater. The detection method is based on the simultaneous recording of the specular reflection and the modification of the speckle pattern from the quantitative speckle contrast is determined. From the specular reflection, we can detect the presence of flat and curved pristine MPs of PET and LDPE. Using the speckle contrast, the presence of MPs in water can also be detected based on the magnitude of the average surface roughness. This study is a step toward the practical implementation of an optical sensor for in situ detection of MPs in an aquatic environment. In the future, it will be interesting to investigate the effect of motion on the detection of low concentration of MPs in water using the dynamic speckle pattern.

Acknowledgements Open access funding provided by University of Eastern Finland (UEF) including Kuopio University Hospital. The authors would like to thank Prof. J. Uozumi for fruitful discussions and suggestions. The work is part of the Academy of Finland Flagship Programme, Photonics Research and Innovation (PREIN), decision 321066.

\section{Compliance with ethical standards}

Conflict of interest On behalf of all authors, the corresponding author states that there is no conflict of interest.

Open Access This article is licensed under a Creative Commons Attribution 4.0 International License, which permits use, sharing, adaptation, distribution and reproduction in any medium or format, as long as you give appropriate credit to the original author(s) and the source, provide a link to the Creative Commons licence, and indicate if changes were made. The images or other third party material in this article are included in the article's Creative Commons licence, unless indicated otherwise in a credit line to the material. If material is not included in the article's Creative Commons licence and your intended use is not permitted by statutory regulation or exceeds the permitted use, you will need to obtain permission directly from the copyright holder. To view a copy of this licence, visit http://creativecommons.org/licenses/by/4.0/. 


\section{References}

1. SAPEA: A scientific perspective on microplastics in nature and society. SAPEA, Berlin (2019)

2. Jambeck, J.R., Geyer, R., Wilcox, C., Siegler, T.R., Perryman, M., Andrady, A., Narayan, R., Law, K.L.: Plastic waste inputs from land into the ocean. Science 348, 768-771 (2015)

3. Zubris, K.A., Richards, B.K.: Synthetic fibers as an indicator of land application of sludge. Environ. Pollut. 138, 201-211 (2005)

4. Rochman, C.M., Tahir, A., Williams, S.L., Baxa, D.V., Lam, R., Miller, J.T., Teh, F.-C., Werorilangi, S., Teh, S.J.: Anthropogenic debris in seafood: Plastic debris and fibers from textiles in fish and bivalves sold for human consumption. Sci. Rep. 5, 1-10 (2015)

5. Zhou, Q., Zhang, H., Fu, C., Zhou, Y., Dai, Z., Li, Y., Tu, C.: The distribution and morphology of microplastics in coastal soils adjacent to the Bohai Sea and the Yellow Sea. Geoderma 322, 201-208 (2018)

6. Pan, Z., Guo, H., Chen, H., Wang, S., Sun, X., Zou, Q., Zhang, Y., Lin, H., Cai, S., Huang, J.: Microplastics in the Northwestern Pacific: Abundance, distribution, and characteristics. Sci. Total Environ. 650(2), 1913-1922 (2019)

7. Ioakeimidis, C., Fotopoulou, K.N., Karapanagioti, H.K., Geraga, M., Zeri, C., Papathanassiou, E., Galgani, F., Papatheodorou, G.: The degradation potential of PET bottles in the marine environment: an ATR-FTIR based approach. Sci Rep 6, 1-8 (2016)

8. Reisser, J., Shaw, J., Hallegraeff, G., Proietti, M., Barnes, D.K.A., Thums, M., Hardesty, B.D., Pattiaratchi, C.: Millimeter-sized marine plastics: a new pelagic habitat for microorganisms and invertebrates. PLoS ONE 9, 1-11 (2014)

9. Hossain, M.R., Jiang, M., Wei, Q., Leff, L.G.: Microplastic surface properties affect bacterial colonization in freshwater. J. Basic Microbiol. 59, 54-61 (2018)

10. Wang, Z., Chena, M., Zhang, L., Wang, K., Yu, X., Zheng, Z., Zheng, R.: Sorption behaviors of phenanthrene on the microplastics identified in a mariculture farm in Xiangshan Bay, Southeastern China. Sci. Total Environ. 628-629, 1617-1626 (2018)

11. Reisser, J., Slat, B., Noble, K., Plessis, K.D., Epp, M., Proietti, M., Sonneville, J.D., Becker, T., Pattiaratchi, C.: The vertical distribution of buoyant plastics at sea: an observational study in the North Atlantic Gyre. Biogeosciences 12, 1249-1256 (2015)

12. Law, K.L., Thompson, R.: Microplastics in the seas. Science 345, 144-145 (2004)

13. Asamoah, B.O., Kanyathare, B., Roussey, M., Peiponen, K.-E.: A prototype of a portable optical sensor for the detection of transparent and translucent microplastics in freshwater. Chemosphere 231, 161-167 (2019)

14. G. Hanke. https://mcc.jrc.ec.europa.eu/documents/2017020740 14.pdf. Accessed Feb 2019. [Online]

15. Julienne, F., Delorme, N., Lagarde, F.: From macroplastics to microplastics: role of water in the fragmentation of polyethylene. Chemosphere 236(124409), 124409 (2019)

16. Peiponen, K.-E., Räty, J., Ishaq, U., Pélisset, S., Ali, R.: Outlook on optical identification of micro- and nanoplastics in aquatic environments. Chemosphere 214, 424-429 (2019)

17. Erf, R.K.: Speckle metrology. Academic, New York (1978)

18. Silvennoinen, R., Peiponen, K.-E., Myller, K.: Specular gloss. Elsevier Science, Amsterdam (2008)

19. Hanson, S.G., Iversen, T.F.Q., Hansen, R.S.: Dynamical properties of speckled speckles. In: Speckle 2010: optical metrology (2010)

20. García, J., Zalevsky, Z., García-Martínez, P., Ferreira, C., Teicher, M., Beiderman, Y.: Projection of speckle patterns for 3D sensing. J. Phys. Conf. Ser. 139, 1-6 (2008)

21. Jain, P., Sarma, S.: Measuring light transport properties using speckle patterns as structured illumination. Sci Rep 9(11157), $1-10$ (2019)

22. Braga, R.A., González-Peña, R.J., Viana, D.C., Rivera, F.P.: Dynamic laser speckle analyzed considering inhomogeneities in the biological sample. J. Biomed. Opt. 22(045010), 045010 (2017)

23. Yamaguchi, I., Yokota, T.I.M., Sunaga, M., Kobayashi, K.: Monitoring of paint drying process by digital speckle correlation. Opt. Rev. 14, 362-364 (2007)

24. Aas, E.: Refractive index of phytoplankton derived from its metabolite composition. J. Plankton Res. 18, 2223-2249 (1996)

25. Lohmann, A., Wirnitzer, B.: Triple correlations. In: IEEE Proceeding (1984)

26. Fujii, H., Asakura, T., Shindo, Y.: Measurements of surface roughness properties by means of laser speckle techniques. Opt. Commun. 16, 68-72 (1976)

27. Ohtsubo, J., Fujii, H., Asakura, T.: Surface roughness measurement by using speckle pattern. Jpn. J. Appl. Phys. 14, 293-298 (1975)

28. Fujii, H., Asakura, T.: Effect of surface roughness on the statistical distribution of image speckle intensity. Opt. Commun. 11, 35-38 (1974)

Publisher's Note Springer Nature remains neutral with regard to jurisdictional claims in published maps and institutional affiliations. 